\title{
Quality and Outcomes Framework: time to take stock
}

We are now living in the seventh annual cycle of the Quality and Outcomes Framework (QOF). So ingrained has QOF become for everyone working in UK primary care, that it is hard to imagine life before QOF. It is hard too, to recall the revolutionary changes that accompanied its arrival. Prior to QOF, there were just two targets for general practice in the UK: childhood immunisations and cervical smears. Suddenly, on 1 April 2004, along came targets for 147 indicators. Initially, 76 of these were clinical targets covering 10 long-term conditions. These were revised in 2006 to 80 targets covering 19 long-term conditions. Implementation occurred within a cultural context which it is important to review when considering future directions for QOF.

The story of the precursors and prerequisites of QOF has already been well told. ${ }^{1}$ In the time leading up to the arrival of QOF, there was public concern about the poor quality of public services, a growing culture of public sector accountability, acceptance that the NHS was underfunded when compared with other European countries, increasing dissemination of information from the evidence-based medicine movement, and a backdrop of economic growth accompanied by the political will to invest in public services.

These factors combined with something of a crisis in primary care: GP recruitment was at an all-time low and a substantial boost to GP income was offered, with QOF as the vehicle. QOF was implemented as a pay-for-performance (P4P) system, structured so that it contained evidencebased indicators and was weighted according to the anticipated workload demands that each indicator would place on an average general practice.

The achievements of QOF have been considerable. It is undoubtedly the most comprehensive, far-reaching P4P system in the world. It has demonstrated high median levels (far higher than originally predicted) of primary care quality achievement, with further annual increases in reported achievement. Consistent improvements have been recorded for 'intermediate outcomes' such as blood pressure, cholesterol, and glycated haemoglobin control. Secondary prevention has been promoted in the form of aspirin for patients with cardiovascular disease, and angiotensin-converting enzyme inhibitors plus beta-blockers for patients with heart failure. Less expected has been the convergence between achievement in prosperous and deprived communities, the shortfall in deprived communities being subsumed by much larger overall gains. ${ }^{2}$ As a result, QOF has been seen as one way of reducing health inequalities, as the targets apply equally to the whole population. Similarly, variability in performance between practices has narrowed. ${ }^{3}$

Many of the successes of QOF are mirrored by concerns about unintended consequences. At first, QOF achievement appeared to show the inferior performance of small practices, particularly singlehanded practices. In their well constructed study, Doran et al have been able to demonstrate rapid improvements in measureable performance by smaller practices, such that by the third year of QOF, practices with fewer than 3000 patients had the highest level of clinical achievement. ${ }^{3}$

Public health goals are dependent on maximum population coverage, and yet the structure of QOF allows GPs to exclude certain patients from QOF targets ('exception reporting') and fails to incentivise achievement beyond pre-defined targets. Most process targets are set at $90 \%$ but intermediate outcome targets such as cholesterol level are set at $60 \%$, blood pressure at $60-70 \%$ (depending on the disease domain), and beta-blockers in heart failure at $60 \%$. Although many practices overshoot the targets, the potential for public health gain appears to be diminished by modest target setting. By highlighting the importance of 'population achievement', Doran et al draw attention to the conflict between the public health and $\mathrm{P} 4 \mathrm{P}$ agendas for QOF. Reconciling this conflict might be achieved by accepting $100 \%$ targets, perhaps accompanied by imaginative tapering and weighting of the rewards according to the difficulty of achievement, particularly for finding and treating the last few patients. Currently, all QOF payment scales are linear but the experience of GPs is more likely to be reflected in a tapered payment system, with most of the financial reward available only for the highest levels of achievement. However a note of caution is needed, as patient feedback has not featured strongly in the evolution of QOF, and $100 \%$ targets may be at odds with patients' right to accept or reject advice from their GP. Although exception reporting has the potential to dilute overall public health gain, it does at least make allowances for patient choice.

QOF is further limited by the perception that it is at odds with the values of professionalism. The Royal College of General Practitioners has recently published their vision, or 'manifesto', of quality: .4 Central to this definition of quality is an emphasis on personal care, longer consultations, continuity of care, and involvement in the healthcare needs of the socially excluded. In contrast, QOF promotes an emphasis on technical excellence. During the consultation itself the very heart of general practice - QOF may be perceived as an unwelcome guest, an intrusive presence representing a pointsdriven, reductionist approach to patient care. ${ }^{5}$ Consultation values such as listening skills, patient enablement, and patient satisfaction do not feature in QOF.

Although QOF could never claim to capture quality in many of these valuesdriven constructs, there is a danger that the success of QOF and its readily understood scoring system could upstage the professional values that are the bedrock of primary care. If we are to see QOF reasserting itself as the means for defining quality, we need a parallel movement to reinforce the professional values of patientcentred care.

Part of the problem of QOF has been its dominance as a measure of quality in 
primary care. And yet quality is a broad concept $^{6}$ and many aspects of quality lie outside QOF. GPs are funded to provide Enhanced Services as part of their contract, targeting a variety of national and local health-related goals, such as substance abuse, alcohol dependency, and minor surgery. Prescribing advisors in most areas set demanding medicines management programmes for promoting prescribing quality.

Many evidence-based indicators are not included in QOF simply because QOF is only designed to reward services that are available nationally. Thus indicators covering interventions of proven effectiveness, such as pulmonary rehabilitation, cardiac rehabilitation, and diabetic educational initiatives, are not incorporated into QOF. Local QOF-based schemes have often appeared to address these shortcomings. One such scheme, 'QOF+', includes indicators covering a diverse range of issues of local importance, such as tuberculosis screening for people arriving from countries of high prevalence, child protection, breast feeding, and alcohol interventions. ${ }^{7}$

Further evidence of the importance of locality factors and the issues about providing high-quality care to socially excluded groups is offered in the paper from Israel by Shani et al, which found particular shortfalls in diabetic targets in older people, new immigrants, and patients living in poorer neighbourhoods, with the implication that care should be focused on these groups. ${ }^{8}$ Quality measurement and accountability is likely to extend to commissioning, and the possibility of a counterpart to QOF termed the Commissioning Outcomes Framework (presumably, 'COF') has been mooted. Alternatively, the organisational domain of QOF could be modified to drive the government agenda of commissioning.

Multiple schemes to promote quality risk fragmenting the concept of quality and there is a need to unify quality initiatives so that each general practice can gauge its overall achievement (summative assessment), plan its quality strategy for the year ahead, build on strengths, and address areas of weakness too (formative assessment). One such unifying concept for quality might be one of the practice accreditation schemes currently being piloted. ${ }^{9}$

QOF was born in an era of plenty, but since the credit crunch in 2007, global recession in 2008, and tax rises planned for 2010 , it is clear that we now need to adapt to an era of public sector austerity. It is timely therefore to consider the article by Fleetcroft et al. ${ }^{10}$ QOF has started to evolve into a value-for-money scheme. Not only should indicators be effective, they should also be cost-effective. This means placing a price on public health gain and offsetting this against the cost of the P4P reward itself. Since the total cost of the QOF scheme is approximately $£ 1$ billion, each QOF point represents $£ 1$ million of public expenditure in England.

The Fleetcroft et al paper highlights the difficulty of costing the benefit of primary care interventions; and sufficiently robust evidence was only found to interpret the impact of 25 clinical indicators. For the first time, these authors have been able to provide an estimate of reduced mortality attributable to QOF achievement. Many assumptions are required for these estimates but the methodology is robust and was based on multiple national data sources to corroborate estimates. The paper also highlights the mismatch between population health gain and QOF points. If the principle of potential for health gain is used as the arbiter for new indicator inclusion in QOF, then this paper has highlighted several new indicators, waiting in the wings.

Perhaps the greatest challenge of all for QOF is the process of continual renewal, ${ }^{11}$ building on its strengths and discarding its less effective components so that QOF fatigue doesn't set in among health professionals and so that more patients benefit from the drive to improve quality. The process is driven by the National Institute for Health and Clinical Excellence (NICE) and it is now possible to glimpse indicators during the course of their development into potential QOF indicators. ${ }^{12}$ Given the 111 indicators currently listed as being at the 'suggestion stage', it would appear that QOF is not short of ideas. Perhaps we should all consider responding to NICE's recent invitation to contribute to suggestions for future QOF topics.

\section{Mark Ashworth}

GP, Hurley Clinic, London, and Clinical Senior Lecturer, Department of Primary Care and Public Health Sciences, King's College London School of Medicine, London.

\section{Maria Kordowicz}

PhD student, Department of Primary Care and Public Health Sciences, King's College London School of Medicine, London.

\section{Provenance}

Commissioned; not peer reviewed.

\section{Competing interests}

The authors have stated that there are none.

\section{REFERENCES}

1. Roland M. Linking physicians' pay to the quality of care - a major experiment in the United Kingdom. $N$ Engl J Med. 2004; 351(14): 1448-1154.

2. Lester H. The UK quality and outcomes framework. BMJ 2008; 337: a2095 [Editorial].

3. Doran T, Campbell S, Fullwood C, et al. The performance of small general practices under the UK's Quality and Outcomes Framework. Br J Gen Pract 2010; 10.3399/bjgp10X515340.

4. Royal College of General Practitioners. Leading the way: high-quality care for all through general practice. London: RCGP, 2010.

http://www.rcgp.org.uk/pdf/RCGP_Political_Manifesto.p df (accessed 29 Jul 2010).

5. Mangin D, Toop L. The Quality and Outcomes Framework: what have you done to yourselves? Br J Gen Pract 2007; 57(539): 435-437.

6. Marshall MN, Campbell S, Hacker J, Roland MO, (eds). Quality indicators for general practice: a practical guide for primary care health professionals and managers. London: Royal Society of Medicine Press, 2002.

7. NHS Hammersmith and Fulham, Imperial College London. Quality and Outcomes Framework Plus: towards world class healthcare for all. London: NHS

Hammersmith and Fulham, Imperial College London, 2008.

http://www.qofplus.org.uk/(S(hiznf155rcldu5eh5eu2pafe ) $/$ GetFile aspx?File=Resources $\% 2 F 0809 \% 2$ FBrief $\% 20$ Int roduction $\% 20$ to\%20QOF\%2B.pdf\&AspxAutoDetectCoo kieSupport=1 (accessed 29 Jul 2010).

8. Shani M, Nakar S, Lustman A, et al. Patient characteristics correlated with quality indicator outcomes 1 in diabetes care. Br J Gen Pract 2010; 60(578): 655-659.

9. Campbell SM, Chauhan U, Lester H. Primary Medical Care Provider Accreditation (PMCPA): pilot evaluation. Br J Gen Pract 2010, 2010; 60(576): e295-e304.

10. Fleetcroft R, Parekh-Bhurke S, Howe A, et al. The UK pay-for-performance programme in primary care: estimation of population mortality reduction. $\mathrm{Br} \mathrm{J} \mathrm{Gen}$ Pract 2010; 10.3399/bigp10X515359.

11. Siriwardena AN. Should the Quality and Outcomes Framework be abolished? BMJ, 2010; 340: c2794.

12. National Institute for Health and Clinical Excellence. About the Quality and Outcomes Framework. London: NICE, 2010.

http://www.nice.org.uk/aboutnice/qof/qof.jsp (accessed 29 July 2010).

DOI: 10.3399/bjgp10X515313

\section{ADDRESS FOR CORRESPONDENCE}

\section{Mark Ashworth}

Department of Primary Care and Public Health Sciences, King's College London School of Medicine, Capital House, 42 Weston Street, London SE1 3QD. E-mail:mark.ashworth@kcl.ac.uk 